\title{
Lipasa endotelial y su relación con la enfermedad cardiovascular y diabetes mellitus tipo 2
}

\author{
CAMILA PIERART Z. ${ }^{1}$, VALENTINA SERRANO L. ${ }^{2}$
}

\section{The role of endothelial lipase in atherogenesis}

Endothelial lipase (EL) is synthetized by endothelial cells and its main substrates are lipoprotein phospholipids. Over expression of EL reduces high density lipoprotein $(H D L)$ cholesterol and phospholipids, in vivo and in vitro. Inhibition of the enzyme achieves the opposite effects. The synthesis of the enzyme is regulated by interleukin 1 and tumor necrosis factor $\alpha$. These inflammatory cytokines play a role in diabetes and vascular disease. An increase in vascular mechanical forces, that play a role in atherogenesis, also increase the synthesis of EL. There is expression of EL in endothelial cells, macrophages and muscle cells of atherosclerotic lesions of coronary arteries of humans. This evidence leads to the suspicion that EL plays a role in atherogenesis. There are also higher plasma levels of EL in subjects with type 2 diabetes, who are especially susceptible to the development of vascular lesions. Therefore the inhibition of EL could play an important role in HDL metabolism and could be a new therapeutic strategy for the prevention of atherosclerosis.

(Rev Med Chile 2012; 140: 373-378).

Key words: Atherosclerosis; Cytokines; Lipase.

L as lipasas son un conjunto de enzimas hidrosolubles que hidrolizan sustratos como los triglicéridos y fosfolípidos, tienen una estructura similar y son fundamentales en el metabolismo de las lipoproteínas y los lípidos. Esta familia está compuesto por la la lipasa pancreática, la lipasa hepática (LH) y la lipasa lipoproteica (LPL) ${ }^{1}$.

En el año 1999 dos grupos describieron, en forma simultánea, un cuarto miembro de esta familia, la lipasa endotelial (LE). Esta nueva enzima se ha relacionado con el proceso de aterogénesis, principalmente por su participación en la modulación de las concentraciones plasmática de colesterol HDL (c-HDL) ${ }^{2,3}$.

LPL y LH son los miembros de la familia de las lipasas mejor caracterizados en la literatura. LPL es sintetizada principalmente por adipocitos, células de músculo esquelético y micardio, mientras que LH es sintetizada por los hepatocitos. Tanto LPL como LH participan en la regulación de los niveles plasmáticos de colesterol y triglicéridos. LPL actúa principalmente sobre triglicéridos hidrolizando lipoproteínas ricas en triglicéridos, mientras que LH tiene actividad sobre triglicéridos y fosfolopidos, hidrolizando LDL y HDL, principalmente las pequeñas con mayor contenido de triglicéridos.

\section{Lipasa endotelial: estructura y síntesis}

LE es una enzima de 482 aminoácidos que se identificó inicialmente en cultivo de células endoteliales de cordón umbilical humano (HUVEC) y en líneas celulares de macrófagos humanos THP-1 expuestos a LDL oxidadas ${ }^{2,3}$.

Esta enzima muestra una secuencia similar a LPL (44\%) y LH (41\%), conservando la secuencia inicial de la señal secretora y el residuo de cisteína implicado en la unión de puentes disulfuro necesarios para la actividad enzimática ${ }^{4}$. La principal diferencia estructural de LE con respecto a los otros miembros de la familia de las lipasas se encuentra 
en el sitio de unión a sustratos ${ }^{2,5}$, formado por 19 residuos aminoacídicos, que corresponde a la región de la proteína que otorga la especificidad de sustrato a la enzima determinando una actividad principalmente sobre fosfolípidos, con un efecto menor sobre triglicéridos ${ }^{2,6}$. Esta característica estructural permite dieferciar el sustrato objetivo en relación a LPL, cuyo sustrato primario son los triglicéridos de los quilomicrones y de las VLDL, y LH que hidroliza triglicéridos y fosfolípidos de prácticamente todas las lipoproteínas, en una proporción similar?

Estudios experimentales han detectado abundante ARN mensajero (ARNm) de LE en células HUVEC y células endoteliales humana de arteria coronaria (HCAEC) ${ }^{2}$. Ensayos de Northern-blot en múltiples tejidos humanos han permitido identificar altos niveles del ARNm de LE en pulmón, hígado, riñón y placenta, que tienen baja expresión de LPL, mientras que no se ha detectado en corazón y músculo esquelético, que expresan abundante LPL ${ }^{2}$. Esta característica es la que le otorga el nombre a la enzima, ya que es sintetizada por las células endoteliales en diferentes tejidos, a diferencia de otras enzimas de esta familia, que actúan en la superficie del endotelio vascular pero son sintetizadas por otros tipos celulares ${ }^{7}$.

En la Tabla 1 se presenta un resumen de las características de LE y la comparación con LPL y LH.

Como se mencionó anteriormente, LE tiene una actividad principalmente de fosfolipasa. Debido a su similitud estructural con las otras lipasas y su actividad de fosfolipasa, se ha propuesto que LE participaría en la hidrólisis de fosfolípidos de las HDL y en la modulación del metabolismo de las HDL in $v i v o^{2,8}$.

Estudios en animales, que utilizaron un adenovirus recombinante que contiene $\mathrm{ADN}$ de $\mathrm{LE}$ humana (AdhLE), demostraron que la sobreex- presión hepática de LE produce una reducción significativa de los niveles plasmáticos de $\mathrm{c}-\mathrm{HDL}^{2,9}$. $\mathrm{Al}$ inyectar el AdhEL a un ratón transgénico para Apo A-I (que tiene niveles plasmáticos muy altos de c-HDL y apo A-1), también se obtuvo una reducción del c-HDL y de apo A- $\mathrm{I}^{2}$. Un estudio realizado en ratones transgénicos que sobreexpresan la enzima $\left(\mathrm{LE}^{+/+}\right)$reportó una disminución de $19 \%$ en los niveles de c-HDL, mientras que en ratones deficientes en $\mathrm{LE}\left(\mathrm{LE}^{-/-}\right)$se encontró un aumento de $25-57 \%$ en los del mismo ${ }^{10}$, apoAI $\mathrm{y}$ apoE ${ }^{11}$. Otro estudio que utilizó un anticuerpo antiLE en ratones, observó un aumento de 25-60\% en los niveles de c-HDL ${ }^{12}$. Estos datos apoyan la hipótesis del importante papel que tendría LE en la regulación de los niveles plasmáticos de c-HDL.

En humanos, se han identificado variantes genéticas de LE en personas con c-HDL eleva$\mathrm{do}^{11,13,14,15}$. En un estudio de 372 personas portadoras de un polimorfismo que determina la reducción de la actividad enzimática, se encontró que los homocigotos para esta mutación tienen un colesterol HDL 14\% mayor que aquellos sujetos que no presentaron la mutación o que resultaron heterocigotos para la misma ${ }^{11}$. Otro estudio realizado en niños sanos en edad escolar, mostró que la presencia de distintos polimorfismos en el gen de LE se asocia a variaciones en los niveles plasmáticos de c-HDL, lo que sugiere que las alteraciones genéticas de esta enzima podrían ser una de las determinantes en las variaciones del c-HDL ${ }^{14}$.

El mecanismo por el cual LE modularía la concentración de c-HDL permanece poco claro. Estudios in vitro han demostrado que LE actuaría remodelando las partículas de HDL, transformándolas en estructuras más pequeñas por pérdida de fosfolípidos, sin que exista disociación de apo $\mathrm{A}-\mathrm{I}^{16}$, lo que la diferencia de $\mathrm{LH}$, que produce una disociación de la apoproteína teniendo esta

Tabla 1. Lipasa endotelial: función y su relación con las HDL

\begin{tabular}{|c|c|c|c|}
\hline & LE & LPL & LH \\
\hline Actividad lipasa & $\mathrm{FL}>\mathrm{TG}$ & $\mathrm{TG}>>>\mathrm{FL}$ & $\mathrm{TG}>\mathrm{FL}$ \\
\hline Sustrato principal & $\mathrm{HDL}$ & Lipoproteínas ricas en TG & Lipoproteínas ricas en $\mathrm{TG}, \mathrm{HDL}$, LDL \\
\hline $\begin{array}{l}\text { Efectos de inflamación sobre la } \\
\text { enzima }\end{array}$ & Aumenta & Disminuye & Disminuye \\
\hline Lugar de síntesis & Célula endotelial & Adipocitos, células musculares & Hepatocito \\
\hline
\end{tabular}

FL: fosfolípidos, TG: triglicéridos, HDL: lipoproteínas de alta densidad, LDL: lipoproteínas de baja densidad. 
última la capacidad de formar nuevas HDL y aceptar colesterol no esterificado y fosfolípidos desde las células periféricas regresando al ciclo del transporte reverso del colesterol ${ }^{17,18}$. También se ha observado que las modificaciones producidas por LE sobre las HDL altera la composición química y física de estas últimas, resultando en una disminución en la capacidad de SR-BI de captarlas y en una disminución del flujo de colesterol SR-BI dependiente ${ }^{19}$.

Estudios in vitro ${ }^{6}$ y en modelos animales ${ }^{2,20}$ sugieren que esta enzima participaría también en la hidrólisis de las partículas lipoproteicas que contienen apoB. La inyección de un AdhLE en un ratón con déficit del receptor de LDL (que tiene aproximadamente $70 \%$ del colesterol en las VLDL/ LDL y $30 \%$ en las HDL) redujo tanto los niveles de c-HDL como de VLDL/LDL, pero el efecto sobre estas últimas fue proporcionalmente menor ${ }^{2}$. No obstante, existe otro estudio en el no se encontró diferencia ni en el número ni en el tamaño de las partículas de LDL en ratones deficientes en $\mathrm{LE}^{21}$, por lo que se necesitan más estudios que permitan llegar a una conclusión en relación a este punto.

En resúmen, los datos aportados por la literatura permiten concluir que LE participa de manera relevante en la modulación de la concentración y metabolismo del colesterol HDL en animales, y que sería un factor importante en la regulación de la concentración plasmática de c-HDL en humanos.

\section{Factores que modulan la actividad de LE}

Estudios realizados en cultivos de células HUVEC y HCAEC han reportado un incremento de 4 veces en los niveles de ARNm de LE en respuesta a la estimulación con IL- $1 \beta$ o TNF $\alpha$, mediado probablemente por activación del factor nuclear $\mathrm{kB}(\mathrm{NFkB})^{22,23}$. Por otra parte, en estos mismos tipos celulares, el efecto de fuerzas físicas, como el estrés por presión, produjo un aumento de 2 a 3 veces en la expresión de ARNm de $\mathrm{LE}^{23}$. Estos datos sugieren que la expresión de LE en células endoteliales es regulada por citoquinas inflamatorias y estímulos físicos, lo que permite centrar la actividad enzimática durante procesos que cursan con algún grado de inflamación y que presentan niveles disminuidos de c-HDL, como la diabetes y la enfermedad cardiovascular.

Por otro lado, se han investigado factores que regulen en forma negativa la expresión de la enzima. Entre estos factores destaca el papel de la actividad física, la que ha demostrado disminuir la actividad enzimática ${ }^{24,25}$ lo que permitiría plantearlo como un mecanismo por el cual el ejercicio causa un aumento en los niveles de cHDL plasmático.

La suplementación con aceite de pescado (rico en ácidos grasos poliinsaturados EPA y DHA) disminuye la actividad de LPL y LH y, probablemente, también la de LE, a través de la inhibición de la producción de IL-6 y TNFa ${ }^{26}$, actuando como reguladores endógenos de las citoquinas proinflamatorias ${ }^{27}$. En presencia de concentraciones adecuadas de EPA/DHA en la célula endotelial, la actividad de la enzima debería disminuir, lo que provocaría un aumento en la concentración de colesterol $\mathrm{HDL}^{28}$.

Estudios clínicos con estatinas han demostrado que estos fármacos producen un aumento de $3-15 \%$ en lo niveles de colesterol HDL y apoA- $\mathrm{I}^{29-32}$, pero existe poca información sobre los mecanismos involucrados en este proceso. Se ha postulado que las estatinas participarían en la modulación de la producción, remodelación y catabolismo periférico de las partículas de $\mathrm{HDL}^{33}$. Por otra parte, existe evidencia de un efecto directo de las estatinas en la inhibición de CEPT ${ }^{34}$. En un estudio realizado en células HUVEC expuestas a Pitavastatina se observó una supresión en la expresión de LE por efecto del fármaco, lo que posteriormente fue confirmado en un estudio en realizado en modelo animal de ratones ${ }^{35}$. Otro estudio en cultivo de macrófagos THP-1 estimulados con Atorvastatina, demostró una disminución de la expresión de LE mediado por una reducción de la activación de NF-kB ${ }^{36}$. Estos datos aportan un nuevo mecanismo, a través de la modulación de LE como efecto del fármaco, que contribuya a explicar como las estatinas producirían un aumento en el colesterol HDL.

\section{Papel de LE en distintas patologías: enfásis en enfermedad cardiovascular y diabetes mellitus tipo 2}

Existe una serie de patologías que se caracterizan por presentar algún grado de inflamación sistémica, entre las que destacan la obesidad, resistencia a la insulina y diabetes tipo 2, hipertensión arteral, dislipidemia y la enfermedad 
cardiovascular ${ }^{37-40}$. En todas estas condiciones es posible encontrar niveles plasmáticos elevados de marcadores inflamatorios, tales como PCR, IL-6 y TNFa, además de una reducción de la concentración plasmática de colesterol HDL ${ }^{39}$. Tal como se ha mencionada anteriormente, estudios in vitro han demostrado que altos niveles de TNF $\alpha$ e IL$1 \beta$ estimulan la expresión de LE en forma dosis dependiente ${ }^{23}$.

Estudios tanto in vivo como in vitro han sugerido que la LE sería un factor adicional en el proceso de aterosclerosis. La sobreexpresión de LE en células endoteliales promueve la adhesión de monocitos a la pared vascular a través de la interacción con los proteoglicanos ${ }^{41}$. Un estudio realizado en ratones deficientes en apo-E sugiere que esta enzima puede modular la aterosclerosis a través de un efecto local a nivel de la pared vascular, reclutando monocitos y aumentando la captación de colesterol ${ }^{42}$. Otro estudio en modelo de ratas hipertensas demostró un aumento de la expresión de LE, sugiriendo que esta enzima tendría algún papel en la patología vascular de esta enfermedad ${ }^{43}$. Por último, se ha detectado la presencia de LE en endotelio de arterias coronarias humanas sanas, y altos niveles de la enzima en arterias coronarias con placas ateroscleróticas avanzadas ${ }^{44,45}$. Sin embargo, Jensen et al no reportaron asociación entre la presencia de una variante genética que inactiva a LE en un grupo de hombres y mujeres caucásicos sanos y los niveles plasmáticos de lípidos o el riesgo de enfermedad coronaria ${ }^{46}$.

Hasta el momento existen escasos estudios en humanos que relacionen LE con alguna patología. En 858 individuos con sobrepeso y antecedentes familiares de enfermedad cardiovascular, se demostró que la concentración plasmática de LE se asocia positivamente con todos los criterios diagnósticos del síndrome metabólico determinados por el NCEP ATP III, y con ateroesclerosis subclínica, medida por el grosor de la media miointimal. Este es el primer estudio que demostró la existencia de una relación negativa entre los niveles plasmáticos de LE y la concentración de c-HDL en humanos, concluyendo que LE actuaría como factor proaterogénico en personas con sobrepeso y síndrome metabólico ${ }^{47}$.

También se ha investigado la asociación que existe entre el tejido adiposo visceral, medido por tomografía axial computada, y la concentración plasmática de LE. En este estudio se observó una correlación positiva entre la cantidad de grasa visceral y los niveles de LE, probablemente, secundario a otras alteraciones metabólicas asociadas a esta condición ${ }^{48}$. Finalmente, se ha demostrado que en humanos existe una correlación directa entre marcadores proinflamatorios (PCRus, IL-6, entre otros) y concentraciones plasmáticas de LE, lo que apoya la hipótesis de que la enzima se encuentra sobre regulada en condiciones de inflamación $\mathrm{n}^{49,50}$.

En un estudio realizado por nuestro grupo en cultivos de células HUVEC estimuladas con distintas concentraciones de glucosa, se observó un bajo nivel basal de LE detectable en la condición control, mientras que al aumentar la concentración de glucosa extracelular los niveles relativos fueron en aumento en forma proporcional, encontrándose una correlación positiva entre la glucosa extracelular y los niveles de $\mathrm{LE}^{51}$. En seres humanos, Shiu et al. mostraron un aumento en la concentración sérica de LE en 237 sujetos con DM2 en comparación con sujetos controles ${ }^{52}$, lo que hemos comprobado en un estudio piloto realizado por nuestro grupo ${ }^{51}$.

En conclusión, LE es una enzima que ha adquirido una importancia creciente en la literatura científica principalmente por su acción en la modulación de la concentración plasmática del c-HDL, permitiendo establecer un nuevo foco de interés para el control, prevención y tratamiento de la enfermedad cardiovascular.

\section{Referencias}

1. Wong H, Schotz M. The lipase gene family. J Lipid Res 2002; 43: 993-9.

2. Jaye M, Lynch KJ, Krawiec J, Marchadier D, Maugeais C, Doan K, et al. A novel endothelial derived lipase that modulates HDL metabolism. Nature genetics 1999; 21: 424-8.

3. Hirata K, Dichek H, Cioffi J, Choi S, Leeper N, Quintana $\mathrm{L}$, et al. Cloning a unique lipase from endothelial cells extends the lipase gene family. J Biol Chem 1999; 274: 1470-5.

4. Murthy V, Julien P, Gagné C. Molecular pathobiology of the human lipoprotein lipase gene. Pharmacol Ther 1996; 70: 101-35.

5. Griffon N, Budreck E, Long C, Broedl U, Marchadier D, Glick J, et al. Substrate specificity of lipoprotein lipase and endothelial lipase: studies of lid chimeras. J Lipid Res 2006; 47: 1803-11.

6. McCoy M, Sun G, Marchadier D, Maugeasis C, Glick 
J, Rader D. Characterization of the lipolytic activity of endothelial lipase. J Lipid Res 2002; 43: 921-9.

7. Hasham S, Pillarisetti S. Vascular lipases, inflammation and atherosclerosis. Clinica Chim Acta 2006; 372: 179183.

8. Annema W, Tietge U. Role of hepatic lipase ande endothelial lipase in high density lipoprotein mediated reverse cholesterol transport. Curr Atheroscler Rep 2011; 13: 257-65.

9. Maugeais C, Tietge U, Broedl U, Marchadier D, Cain W, McCoy M, et al. Dose dependent acceleration of high density lipoprotein catabolism by endothelial lipase. Circulation 2003; 108: 2121-6.

10. Ishida T, Choi S, Kundu RK, Hirata K, Rubin E, Cooper A, et al. Endothelial lipase is a major determinant of HDL level. J Clin Invest 2003; 111: 347-55.

11. Ma K, Cilingiroglu M, Otvos J, Ballantyne C, Marian A, Chan L. Endothelial lipase is a major genetic determinant for high density lipoprotein concentration, structure and metabolism. Proc Natl Acad Sci USA 2003; 100: 2748-53.

12. Jin W, Millar J, Broedl U, Glick J, Rader D. Inhibition of endothelial lipase causes increased HDL cholesterol levels in vivo. J Clin Invest 2003; 111:357-62.

13. Delmos A, Wolfe M, Long C, Sivapackianathan R, Rader D. Identification of genetic variants in Endothelial lipase in persons with elevated high density lipoprotein cholesterol. Circulation 2002; 106: 1321-6.

14. Yamakawa-Kobayashi K, Yanagi H, Endo K, Arinami T, Hamaguchi H. Relationship between serum HDL-C levels and common genetic variants of the endothelial lipase gene in Japanese school-aged children. Human Genet 2003; 113: 311-5.

15. Brown R, Edmondson A, Griffon N, Hill T, Fuki I, Badellino $\mathrm{K}$, et al. A naturally occurring variant of endothelial lipase associated with elevated HDL exhibits impaired synthesis. J Lipid Res 2009; 50: 1910-6.

16. Jahangiri A, Rader D, Marchadier D, Curtiss L, Bonnet D, Rye K. Evidence that endothelial lipase remodels high density lipoproteins without mediating the dissociation of lipoprotein A-I. J Lipid Res 2005; 46: 896-903.

17. Clay M, Rye K, Barter P. Evidence in vitro that hepatic lipase reduces the concentration of apolipoprotein A-I in rabbit high density lipoproteins. Biochim Biophys Acta 1990; 1044: 50-6.

18. Clay M, Newmhan H, Barter P. Hepatic lipase promotes a loss of apolipoprotein A-I from triglyceride enriched human high density lipoproteins during incubation in vitro. Arterioscler Thromb 1991; 11: 415-22.

19. Gauster M, Oskolkova O, Innerlohinger J, Glatter O, Knipping G, Frank S. Endothelial lipase modified high density lipoprotein exhibits diminished ability to mediated SR-BI (scavenger receptor type I) dependent free cholesterol efflux. Biochem J 2004; 382: 75-82.

20. Broedl U, Maugeais C, Millar J, Weijun J, Moore R, Fuki I, et al. Endothelial lipase promotes the catabolism of Apo-B containing lipoproteins. Cir Res 2004; 94: 155461.

21. Ma K, Forte T, Otvos J, Chan L. Differential additive effects of endothelial lipase and scavenger receptor class B type I on high density lipoprotein metabolism in knockout mouse models. Arterioscler Throm Vasc Biol 2005; 25: 149-54.

22. Hirata K, Ishida T, Matsushita H, Tsao P, Quertermous T. Regulated expression of endothelial cell derived lipase. Biochem Biophys Res Commun 2000; 272: 90-3.

23. Jin W, Sun G, Machadier D, Octtaviani E, Glick J, Rader D. Endothelial cells secrete triglyceride lipase and phospholipase activities in response to cytokines as a result of endothelial lipase. Cir Res 2003; 92: 644-50.

24. Halverstdt A, Phares D, Ferrel R, Wilund K, Goldberg A, Hagberg J. High density lipoprotein cholesterol, its subfraction and responses to exercise training are dependent on endothelial lipase genotype. Matabolism 2003; 52: 1505-11.

25. Smith CE, Arnett DK, Tsai MY, Lai CQ, Parnell LD, Shen $\mathrm{J}$, et al. Physical inactivity interacts with an endothelial lipase polymorphism to modulate high density lipoprotein cholesterol in the GOLDN study. Atherosclerosis 2009; 206: 500-4.

26. Haug A, Hostmark A. Lipoprotein lipases, lipoproteins and tissue lipid in rats fed fish oil or coconut oil. J Nutr 1987; 117: 1011-7.

27. Das U. Benefical effects of n-3 fatty acids in cardiovascular disease: but why and how? Prostaglandins Leukot Essent Fatty Acids 2000; 63: 351-62.

28. Das U. Long chain polyunsatured fatty acids, endothelial lipase and atherosclerosis. Prostaglandins Leukot Essent Fatty Acids 2005; 72: 173-9.

29. Ballantyne C, Olsson A, Cook T, Mercuri M, Pedersen T, Kjekshus J. Influence of low high-density lipoprotein cholesterol and elevated triglyceride on coronary heart disease events and response to simvastatin therapy in $4 \mathrm{~S}$. Circulation 2001; 104: 3046-51.

30. Streja L, Packard CJ, Shepherd J, Cobbe S, Ford I. Factors affecting low-density lipoprotein and high-density lipoprotein cholesterol response to pravastatin in the West of Scotland coronary prevention study (WOSCOPS). Am J Cardiol 2002; 90: 731-6.

31. National Cholesterol Education Panel: Third Report of the National Cholesterol Education Program Expert Panel on Detection, Evaluation and Treatment of High 
Blood Cholesteron in Adults final report. Circulation 2002; 106: 3143-421.

32. Ridker P, Damelson E, Fonseca F, Grust J, Gotto A, Kastelein J, et al, for the JUPITER study group. Rosuvastatin to prevent cardiovascular events in men and woman with elevated C reactive protein. N Engl J Med 2008; 359: 2195-207.

33. Uno K, Nicholls S. Status effects on both kow density lipoproteins and high density lipoproteins. Is there a dual benefit?. Curr Athersocler Rep 2010; 21: 14-9.

34. Davidson M, Toth P. High-density lipoprotein metabolism: potential therapeutic targets. Am J Cardiol 2007; 100: $32 \mathrm{~N}-40 \mathrm{~N}$.

35. Kojima Y, Ishida T, Sun L, Yasuda T, Toh R, Rikitake Y, et al. Pitavastatin decreases expression of endothelial lipase both in vitro and in vivo. Cardiovasc Res 2010; 15: 38593.

36. Qiu G, Hill J. Atorvastatin decrease lipoprotein lipase and endothelial lipase expression in human THP-1 macrophages. J Lipid Res 2007; 48: 2112-22.

37. Ross R. Atherosclerosis an inflammatory disease. N Engl J Med 1999; 340; 115-26.

38. Das $\mathrm{U}$. Is metabolic syndrome $\mathrm{X}$ an inflammatory condition?. Exp Biol Med 2002; 227: 989-97.

39. Lyon C, Law R, Hshueh W. Adiposity, inflammation and atherogenesis. Endocrinology 2003; 144: 2195-2000.

40. Sowers J, Stump C. Insights into the biology of diabetic vascular disease: what's new?. Am J Hypertens 2004; 17: 2S-6S.

41. Kojma $Y$, Hirata K, Ishida T, Shimokawa $Y$, Inoue N, Kawashima S, et al. Endothelial lipase modulates monocytes adhesion to the vessel wall. A potential role in inflammation. J Biol Chem 2004; 279: 54032-8.

42. Ishida T, Choi S, Kundu R, Spin J, Yamashita T, Hirata $\mathrm{K}$, et al. Endothelial lipase modulates susceptibility to atherosclerosis in apolipoprtein E deficient mice. J Bio
Chem 2004; 43: 45085-92.

43. Shimokawa Y, Hirata K, Ishida T, Kojma Y, Inoue N, Quertermous T, et al. Increased expression of endothelial lipase in rat models of hypertension. Cardiovasc Res 2005; 66: 594-600.

44. Azumi H, Hirata K, Ishida T, Kojima Y, Rikitake Y, Takeuchi S, et al. Immunohistochemical localization of endothelial cell derived lipase in atherosclerotic human coronary arteries. Cardiovasc Res 2003; 58: 647-54.

45. Bartels E, Nielsen J, Lindegaard M, Hulten 1, Schroeder T, Nielsen L. Endothelial lipase is highly expressed in macrophages in advanced human atherosclerotic lesions. Atherosclerosis 2007; 195: e42-9.

46. Jensen M, Rimm E, Mukamal M, Edmonson A, Rader $\mathrm{D}$, Vogel $\mathrm{U}$, et al. The T111I variant in the endothelial lipase gene and risk of coronary heart disease in three independent populations. Eur Heart J 2009; 30: 1584-9.

47. Badellino K, Wolfe M, Reilly M, Rader D. Endothelial lipase concentrations are increased in metabolic syndrome and associated with coronary atherosclerosis. PLoS Medicine 2006; 3: 245-52.

48. Paradis M, Badellino K, Rader D, Tchamof A, Richard C, Luu-The V, et al. Visceral adiposity and endothelial lipase. J Clin Endocrinol Metab 2006; 91: 3538-43.

49. Paradis M, Badellino, K, Rader D, Deshaies Y, Couture P, Archer W, et al. Endothelial lipase is associated with inflammation in humans. J Lipid Res 2006; 47: 2808-13.

50. Badellino K, Wolfe M, Reilly M, Rader D. Endothelial lipase is increased in vivo by inflammation in humans. Circulation 2008; 117: 678-85.

51. Pierart C, Serrano V, Rubio L, Ebensperger R, Foncea R. Efecto de glucose en la expression de lipasa endothelial en células endoteliales humanas y en sujetos con diabetes mellitus tipo 2. Nutr Hosp 2001; 26: 915-20.

52. Shiu S, Tan K, Huang Y, Wong Y. Type 2 diabetes mellitus and endothelial lipase. Atherosclerosis 2008; 198: 441-7. 